(C) 2017 IEEE. Personal use of this material is permitted. Permission from IEEE must be obtained for all other uses, in any current or future media, including reprinting/republishing this material for advertising or promotional purposes, creating new collective works, for resale or redistribution to servers or lists, or reuse of any copyrighted component of this work in other works. 


\title{
Multistep Model Predictive Control for Cascaded H-Bridge Inverters: Formulation and Analysis
}

\author{
Roky Baidya, Student Member, IEEE, Ricardo P. Aguilera, Member, IEEE, Pablo Acuña, Member, IEEE, \\ Sergio Vazquez, Senior Member, IEEE, Hendrik du Toit Mouton, Member, IEEE
}

\begin{abstract}
In this work, a suitable long prediction horizon (multistep) model predictive control (MPC) formulation for cascaded H-bridge inverters is proposed. The MPC is formulated to include the full steady-state system information in terms of output current and output voltage references. Generally, basic single-step predictive controllers only track the current references. As a distinctive feature, the proposed MPC also tracks the control input references, which in this case is designed to minimize the common-mode voltage (CMV). This allows the controller to address both output current and CMV targets in a single optimization. To reduce the computational effort introduced by a long prediction horizon implementation, the proposed MPC formulation is transformed into an equivalent optimization problem that can be solved by a fast sphere decoding algorithm. Moreover, the benefits of including the control input references in the proposed formulation are analyzed based on this equivalent optimization problem. This analysis is key to understand how the proposed MPC formulation can handle both control targets. Experimental results show that the proposal provides an improved steady-state performance in terms of current distortion, inverter voltages symmetry, and CMV.
\end{abstract}

Index Terms-Multilevel converters, cascaded H-bridge, common-mode voltage, finite control set, model predictive control, optimization problem, sphere decoding.

\section{INTRODUCTION}

$\mathbf{M}$ ULTILEVEL converters (MCs) have been the preferred commercial alternative for medium/high-power applications, such as industrial motor drives, transmission systems, active power filters and renewable energy conversion [1]. Nowadays, there exist three popular topologies of MCs: flying capacitors (FC) [2], neutral point clamped (NPC) [3] and cascaded H-bridge (CHB) [4]. Among these topologies, the $\mathrm{CHB}$ converter has emerged as a prominent one due to its high degree of modularity, which allows the converter to reach high voltages and currents using medium-voltage semiconductor devices. Despite the fact that this CHB topology has been available for decades, there is still an undergoing research mainly focused on improving modulation techniques and control strategies used [4], [5].

Roky Baidya and Pablo Acuña are with the School of Electrical Engineering and Telecommunications, The University of New South Wales, Sydney, NSW 2052, Australia (e-mails: roky.baidya@unsw.edu.au; pablo.acuna@unsw.edu.au).

Ricardo P. Aguilera is with the School of Electrical, Mechanical and Mechatronic Systems, University of Technology Sydney, Sydney, NSW 2007, Australia (e-mail: raguilera@ieee.org).

S. Vazquez is with the Department of Electronic Engineering, Universidad de Sevilla, 41092 Seville, Spain (e-mail: sergi@us.es).

H. du T. Mouton is with the Department of Electrical and Electronic Engineering, University of Stellenbosch, Private Bag X1, Matieland 7602, South Africa (e-mail: dtmouton@sun.ac.za).
Regarding the control of power converters, model predictive control (MPC) has emerged as an attractive alternative to classical control methods [6], [7]. The potentiality of MPC comes from its ability to handle multi-variable systems, nonlinearities, and system constraints. Among the MPC families, the finite-control-set MPC (FCS-MPC) is one of the most promising strategies for power converters [7]. FCS-MPC directly considers the switch states (or voltage levels) as control inputs into the optimization problem. Some examples of recent predictive control formulations in power electronics can be found in [8]-[12].

In general, a predictive controller can be divided into two main stages. The first one is the optimal control formulation. Here, different control targets are incorporated into a cost function that forecasts the system behavior several time-steps (multistep) ahead by considering a long prediction horizon. These control targets can be the standard system-state references, e.g., current, voltages, power, torque, etc., and also the ones related to the converter itself, e.g., reduced switch commutations, common-mode voltage (CMV) minimization, internal capacitor balancing, etc. The optimal control formulation stage can also include the so-called, reference design step in order to further improve the closed-loop behavior; see e.g., [8], [13], [14] where a suitable dynamic reference for the dc-link voltage is designed in order to control both ac-and dc-side variables of an active front-end rectifier. The second stage is the optimization process. Normally, for FCSMPC strategies one can obtain the optimal control input (OCI), by simply evaluating the cost function for all possible input combinations, which is known as exhaustive search algorithm (ESA). Therefore, the input combination which gives the minimum cost value is, thus, the OCI.

An on-line implementation of FCS-MPC using ESA is relatively simple, which is another reason for its popularity. However, if the optimal problem presents a large number of input combinations, then the required computational effort to evaluate all the possible combinations might exceed the microcontroller capabilities. This restricts the ESA-based FCSMPC applicability to MCs with few power switches and its formulation to single-step cost functions [6], [7].

Recently, a single-step FCS-MPC strategy for CHB converters was presented in [15]. Here, the problem is formulated in the $\alpha \beta$-framework. Thus, the number of control inputs can be reduced by discarding the redundant vectors. To further reduce the input combinations to be explored by the ESA, in [15] the search space is limited to a subset formed by the vectors that are adjacent to the previous optimal input vector. 
Despite the benefit of the reduction in the required calculation time to obtain the OCI, this basic single-step predictive current controller can lead to a high CMV since it is only formulated to consider the current tracking error. Mitigation of CMV in medium/high power applications is important to enhance the reliability of the system ${ }^{1}$ (preventing the premature failure of motor insulations, bearing currents, electromagnetic interference problems and ground faults) [17]-[19]. To address this, in [15] a secondary optimization is considered which reduces the resulting CMV. It is for this reason that in [15], redundant vectors are reduced by choosing only the ones that produce minimum CMV. Nevertheless, it can lead to an asymmetry in the inverter voltages. In [20], a simplified MPC with CMV reduction capability is proposed. Here, a dead-beat based approach is firstly used to obtained an output voltage reference. Thus, the optimal input is the inverter voltage vector which is nearest this reference. Therefore, current predictions are no required. Moreover, to account for the CMV, an extra term to the cost function which penalizes the CMV can be added, in a similar manner as in [21]. Even though these approaches can effectively reduce the $\mathrm{CMV}$, they rely on an ESA to find the OCI. This impedes the implementation of these approaches in MCs with large output voltage levels and/or long prediction horizon formulations.

On the other hand, it has been recently shown that MPC with long prediction horizon can significantly improve the resulting steady-state performance (e.g., lowering the total harmonic distortion (THD) of output currents, switching frequency reduction, etc) when compared to the basic single-step MPCs [22]. Particularly in [23], [24], a sphere decoding algorithm (SDA) has been proposed for the optimization process, which is a fast and efficient alternative to ESA.

In this work, a long prediction horizon FCS-MPC formulation for $\mathrm{CHB}$ inverters that considers the full steady-state system information in terms of output currents and voltages is proposed. Here, the controller is designed based on the formal MPC formulation used in control theory, where the cost function is built to track both system state and control input references [25]. In the work at hand, the three-phase output currents are chosen as system states while the output voltage levels are considered as control inputs. Normally, a standard FCS-MPC is formulated only to track a threephase sinusoidal current reference. However, as a distinctive feature, the proposed FCS-MPC also tracks the control input references which are designed to minimize the CMV. To do this, the predictive controller is formulated in the original $a b c$-framework, which allows the system model to retain the CMV information. To account for the computational burden introduced by a long prediction horizon implementation, the optimization problem is transformed into an equivalent triangular integer least squares (ILS)-problem which can be solved by an efficient SDA. This is possible thanks to the formal MPC formulation used in this work. This paper extends the preliminary work [26] by graphically analyzing the ILSproblem of the proposed FCS-MPC. This analysis is key to

${ }^{1}$ In some applications, injection of CMV is desired to account for unbalanced power generation levels, e.g., in large PV solar plants [16].

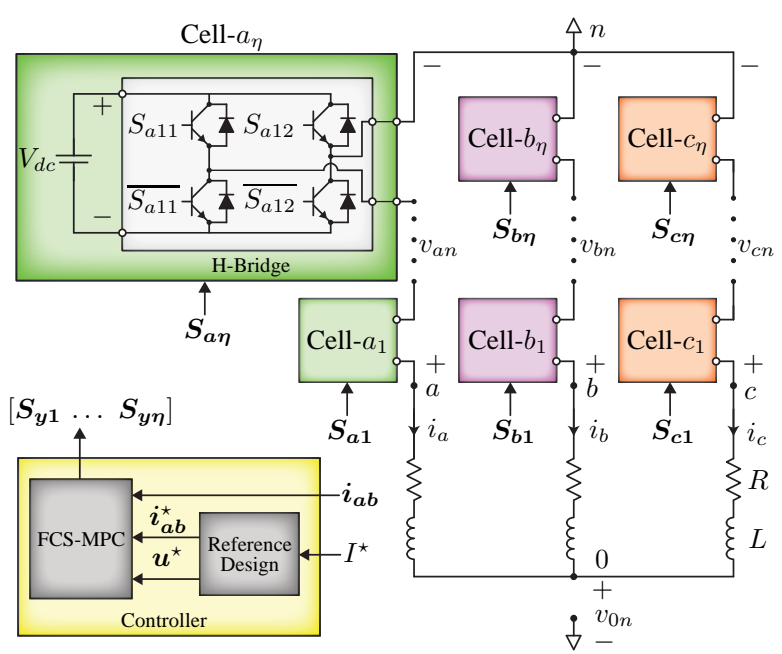

Fig. 1. Circuit schematic of a three-phase $\eta$-cell CHB inverter with passive $R L$ loads and block diagram of the proposed control scheme.

understand how the controller can achieve a desired steadystate performance by tracking both the system states and control input references. As evidenced by the experimental results, the steady-state performance provided by the proposed FCSMPC with horizon-one outperforms the one obtained with the basic single-step FCS-MPC. Moreover, further improvements are achieved as the prediction horizon is increased. On the other hand, the resulting dynamic performance of the proposed control strategy with long prediction horizon preserves the inherent fast dynamic response of the basic single-step FCSMPC.

\section{STANDARD FCS-MPC OF A CHB INVERTER}

This section describes a generalized CHB inverter system model and presents a common procedure used to apply a standard FCS-MPC problem formulation when a basic singlestep cost function based on [7] is considered.

\section{A. CHB Inverter Model}

A three-phase CHB inverter with $\eta$-cell per phase which supplies power to passive RL loads is shown in Fig. 1. Here, each cell is a 3-level H-bridge converter which is electrically fed with an isolated dc voltage source. The addition of each cell output voltage in a phase produces the total output voltage, $v_{y n}(t)$. Thus, the continuous-time dynamic model of a CHB inverter can be written as:

$$
\frac{d i_{y}(t)}{d t}=-\frac{R}{L} i_{y}(t)+\frac{1}{L}\left(v_{y n}(t)-v_{0 n}(t)\right),
$$

for all $y \in\{a, b, c\}$ and

$$
v_{y n}(t)=\sum_{j=1}^{\eta} v_{y j}(t),
$$

where $v_{y j}(t)$ is the individual cell output voltage and $\eta$ denotes the number of cells per phase. Furthermore, the CMV, $v_{0 n}(t)$, is given by:

$$
v_{0 n}(t)=\frac{1}{3}\left(v_{a n}(t)+v_{b n}(t)+v_{c n}(t)\right)
$$




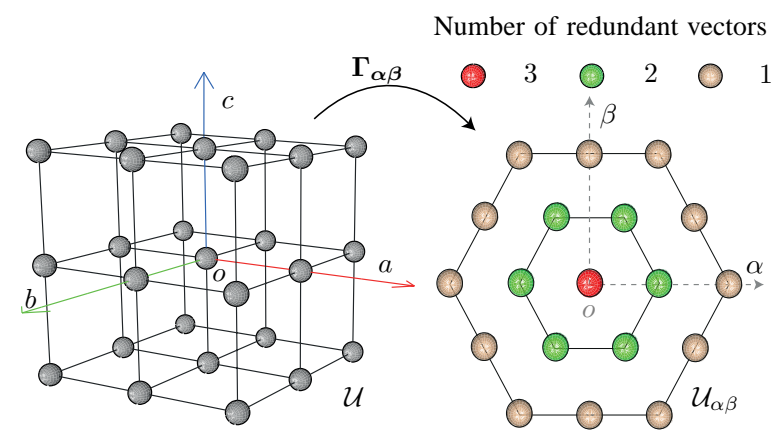

Fig. 2. Clarke's transformation, $\boldsymbol{\Gamma}_{\boldsymbol{\alpha} \boldsymbol{\beta}}$, of control inputs in $a b c$-framework to $\alpha \beta$-framework for a three-phase one-cell $(\eta=1) \mathrm{CHB}$ converter.

which holds for three-phase three-wire balanced/unbalanced systems, where both load and converter neutral points are not connected.

In general, an FCS-MPC governing a power converter considers the states of power switches as control input [27]. This work considers the phase voltage levels, $v_{\ell y}$, as the control input, which significantly reduces the number of input combinations compared to the states of the power switches. Then, the total output voltage becomes:

$$
v_{y n}=V_{d c} v_{\ell y}
$$

where

$$
v_{\ell y} \in \mathbb{V}=\{-\eta,-\eta+1, \ldots, 0, \ldots, \eta-1, \eta\}
$$

Hence, for a three-phase $\eta$-cell CHB converter, the number of voltage level combinations considering (5) is given by:

$$
N_{V L C}=(2 \eta+1)^{3} \text {. }
$$

For each voltage level combination, the states of the power switches, $\boldsymbol{S}_{\boldsymbol{y} \boldsymbol{j}}, \forall j \in[1, \eta]$, are obtained by applying a Sorting Algorithm (SA) which aims for equal utilization of power switches, cf. [28], [29] where SA is used for capacitor voltage balancing and power losses distribution.

At any discrete-time instant $k$, the system states and control inputs are chosen as:

$$
\boldsymbol{i}_{\boldsymbol{a} \boldsymbol{b}}(k)=\left[\begin{array}{c}
i_{a}(k) \\
i_{b}(k)
\end{array}\right] \in \mathbb{R}^{2}, \boldsymbol{u}(k)=\left[\begin{array}{c}
v_{\ell a}(k) \\
v_{\ell b}(k) \\
v_{\ell c}(k)
\end{array}\right] \in \mathcal{U}=\mathbb{V}^{3},
$$

where $i_{c}(k)=-\left(i_{a}(k)+i_{b}(k)\right)$ and the input, $\boldsymbol{u}(k)$, belongs to the FCS, $\mathcal{U}=\mathbb{V}^{3}$. By applying the forward Euler discretization to (1) with a sampling period of $T_{s}$, the following discrete-time dynamic model can be obtained:

$$
\boldsymbol{i}_{\boldsymbol{a} \boldsymbol{b}}(k+1)=\boldsymbol{A} \boldsymbol{i}_{\boldsymbol{a} \boldsymbol{b}}(k)+\boldsymbol{B} \boldsymbol{u}(k),
$$

where

$$
\boldsymbol{A}=\left[\begin{array}{cc}
1-\frac{R T_{s}}{L} & 0 \\
0 & 1-\frac{R T_{s}}{L}
\end{array}\right], \boldsymbol{B}=\frac{V_{d c} T_{s}}{3 L}\left[\begin{array}{ccc}
2 & -1 & -1 \\
-1 & 2 & -1
\end{array}\right] .
$$

\section{B. Standard Problem Formulation}

Generally, the standard FCS-MPC strategy is formulated with a cost function that considers the control targets over one-step prediction horizon based on the system model [6], [7]. At each discrete instant $k$, the cost function is evaluated for each element of the FCS $\mathcal{U}$ to obtain an OCI to be applied to the converter. If a basic single-step cost function that only considers the output currents tracking error is used, then the control target is to maintain a sinusoidal steady-state references for these output currents, i.e.:

$$
\boldsymbol{i}_{\boldsymbol{a} \boldsymbol{b}}^{\star}(k)=\left[\begin{array}{c}
I^{\star} \sin \left(\omega k T_{s}\right) \\
I^{\star} \sin \left(\omega k T_{s}-2 \pi / 3\right)
\end{array}\right],
$$

where $i_{a b}^{\star}$ stands for the output current references, in which $I^{\star}$ is the peak value. Thus, a basic single-step cost function in the original stationary $a b c$-framework can be expressed as (see [7]):

$$
J_{a b c}=\left\|\boldsymbol{i}_{\boldsymbol{a b}}(k+1)-\boldsymbol{i}_{\boldsymbol{a} \boldsymbol{b}}^{\star}(k+1)\right\|_{2}^{2},
$$

where $\boldsymbol{i}_{\boldsymbol{a} \boldsymbol{b}}(k+1)$ stands for the current predictions based on the system model (8) and $\boldsymbol{i}_{\boldsymbol{a} \boldsymbol{b}}^{\star}(k+1)$ is the next step current references. Here, the FCS $\mathcal{U}$ presents redundant elements in terms of the output current, i.e., there is more than one control input that can produce the same output current. Alternatively, the control problem can be formulated in the stationary orthogonal $\alpha \beta$-framework [15]. Then, the cost function (11) after applying the well-known Clarke's transformation, $\boldsymbol{\Gamma}_{\boldsymbol{\alpha} \boldsymbol{\beta}}$, over $\mathcal{U}$, i.e., $\mathcal{U}_{\alpha \beta}=\boldsymbol{\Gamma}_{\boldsymbol{\alpha} \boldsymbol{\beta}} \cdot \mathcal{U}$, becomes:

$$
J_{\alpha \beta}=\left\|\boldsymbol{i}_{\boldsymbol{\alpha} \boldsymbol{\beta}}(k+1)-\boldsymbol{i}_{\boldsymbol{\alpha} \boldsymbol{\beta}}^{\star}(k+1)\right\|_{2}^{2},
$$

where $\boldsymbol{i}_{\boldsymbol{\alpha} \boldsymbol{\beta}}, \boldsymbol{i}_{\boldsymbol{\alpha} \boldsymbol{\beta}}^{\star}$ are the current predictions and references in $\alpha \beta$-framework, respectively. This formulation allows one to reduce the number of elements in $\mathcal{U}$ by discarding the redundant inputs. For instance, in a three-phase one-cell $(\eta=1)$ CHB converter, the FCS $\mathcal{U}=\mathbb{V}^{3}=\{-1,0,1\}^{3}$ comprises 27 -input vectors as per (6), and forms a cube-lattice in the $a b c$ framework, as shown in Fig. 2. Thus, performing the Clarke's transformation $\Gamma_{\boldsymbol{\alpha} \beta}$ on $\mathcal{U}$, yields a new reduced FCS $\mathcal{U}_{\alpha \beta}$ which forms a hexagon with only 19-distinct vectors in the $\alpha \beta$ framework. It is important to emphasize that both formulations (11) and (12) are equivalent in terms of optimality albeit the latter reduces the required computations performed by the MPC algorithm.

\section{PROPOSED FCS-MPC FORMULATION}

Motivated by the issues discussed in the introduction, this work proposes a long prediction horizon FCS-MPC formulation for $\mathrm{CHB}$ inverters that considers the full steady-state system information in terms of output currents and voltages. Here, the controller is designed based on the formal MPC formulation used in control theory [30], where the cost function is built to track both system state and control input references $^{2}$. A key feature of this work, compared to the basic single-step FCS-MPC, is that the proposed controller

\footnotetext{
${ }^{2}$ This formal MPC formulation also allows one to study stability and robustness of predictive controllers for power converters [30]-[34].
} 
also tracks the control input references, which are designed to minimize the CMV. This is achieved by formulating the optimal control problem in the original $a b c$-framework, where the CMV information is preserved through the matrix $\boldsymbol{B}$ in (9). Therefore, the proposed cost function for an arbitrary prediction horizon, $N$, becomes:

$$
J_{N}=\sum_{l=k}^{k+N-1}\left\|\boldsymbol{i}_{\boldsymbol{a} \boldsymbol{b}}(l+1)-\boldsymbol{i}_{\boldsymbol{a} \boldsymbol{b}}^{\star}(l+1)\right\|_{2}^{2}+\sigma\left\|\boldsymbol{u}(l)-\boldsymbol{u}^{\star}(l)\right\|_{2}^{2},
$$

where $\boldsymbol{u}(k)$ is the tentative control input that generates the current prediction $\boldsymbol{i}_{\boldsymbol{a} \boldsymbol{b}}(k+1)$, and $\boldsymbol{u}^{\star}(k)$ stands for the required CHB output voltages to maintain the current references (10) in steady-state, i.e.:

$$
\begin{aligned}
\boldsymbol{u}^{\star}(k) & =\left[\begin{array}{lll}
v_{\ell a}^{\star}(k) & v_{\ell b}^{\star}(k) & v_{\ell c}^{\star}(k)
\end{array}\right]^{T} \in \mathbb{R}^{3}, \\
v_{\ell y}^{\star}(k) & =\frac{I^{\star}}{V_{d c}}\left(\omega L \cos \left(\omega k T_{s}+\phi_{y}\right)+\frac{R}{V_{d c}} \sin \left(\omega k T_{s}+\phi_{y}\right) .\right.
\end{aligned}
$$

In (14), $v_{\ell y}^{\star}(k)$ is derived by using (1) and (10), while considering a null CMV (i.e., $v_{0 n}=0$ ). Moreover, $\phi_{a}=0$, $\phi_{b}=-2 \pi / 3$ and $\phi_{c}=2 \pi / 3$. The weighting factor $\sigma$ in (13) penalizes the impact of the control input references tracking by regulating a desired closed-loop response, see [31].

Since the proposed formulation considers $N>1$, it is convenient to introduce the input sequence $\boldsymbol{U}(k)$ denoted by:

$$
\boldsymbol{U}(k)=\left[\boldsymbol{u}^{T}(k) \ldots \boldsymbol{u}^{T}(k+N-1)\right]^{T} \in \mathbb{U} \subset \mathbb{R}^{3 N},
$$

where the FCS $\mathbb{U}=\mathcal{U}^{N}=\mathbb{V}^{3 N}$. Thus, the optimization problem directing to FCS-MPC strategy can be formulated as:

$$
\begin{aligned}
\boldsymbol{U}_{\mathrm{opt}}(k) & =\arg \left\{\min _{\boldsymbol{U}(k)} J_{N}\right\}, \\
\text { subject to: } \quad \boldsymbol{i}_{\boldsymbol{a} \boldsymbol{b}}(l+1) & =\boldsymbol{A} \boldsymbol{i}_{\boldsymbol{a} \boldsymbol{b}}(l)+\boldsymbol{B} \boldsymbol{u}(l), \\
u(l) & \in \mathcal{U}, \\
\|\Delta \boldsymbol{u}(l)\|_{\infty} & \leq 1,
\end{aligned}
$$

for all $l \in\{k, \ldots, k+N-1\}$, and where $\Delta u(l)=$ $u(l-1)-u(l)$. Here, (16b)-(16d) encompasses the physical power converter constraints, where: (16b) refers to the system constraint given by the inverter model (8); (16c) is the FCS $\mathcal{U} \in \mathbb{V}^{3}$ constraint for the tentative input, $\boldsymbol{u}(l)$, given by the output voltage levels; and (16d) is the inverter phase voltage level constraint. The latter takes into account the fact that one step voltage level change is recommended to avoid high $d v / d t$ rating in medium/high power converters.

Consequently, the long prediction horizon FCS-MPC is formulated to achieve balanced sinusoidal currents with a reduced CMV by solving the optimization problem (16a). This results in the optimal input sequence:

$$
\boldsymbol{U}_{\mathrm{opt}}(k)=\left[\begin{array}{lll}
\boldsymbol{u}_{\mathrm{opt}}^{T}(k) & \ldots & \boldsymbol{u}_{\mathrm{opt}}^{T}(k+N-1)
\end{array}\right]^{T},
$$

which minimizes the cost function (13). Following the receding horizon policy, at each sampling instant only the first element of $\boldsymbol{U}_{\text {opt }}(k)$ (i.e., $\boldsymbol{u}_{\text {opt }}(k)$ ) is applied to the converter discarding the remaining ones. Therefore, the resulting closedloop system equation for the $\mathrm{CHB}$ converter becomes:

$$
\boldsymbol{i}_{\boldsymbol{a} \boldsymbol{b}}(k+1)=\boldsymbol{A} \boldsymbol{i}_{\boldsymbol{a b}}(k)+\boldsymbol{B} \boldsymbol{u}_{\mathrm{opt}}(k) .
$$

Notice that as $N$ and $\eta$ increase, a high computational effort is required for the ESA when solving the optimization problem (16). As a consequence, a fast optimization algorithm is needed to reduce the computational complexity.

\section{OPTIMIZATION PROCESS}

The control problem in (16) requires a suitable formulation that facilitates the long prediction horizon implementation. Thus, the SDA described in [23], [35], [36] is adopted as a computationally efficient optimizer.

\section{A. Optimization Problem Reformulation}

At first, the cost function (13) is presented as a function of input sequence $\boldsymbol{U}(k)$ by successively using the system model (16b) for all $l \in\{k, \ldots, k+N-1\}$, i.e.:

$$
J_{N}=\boldsymbol{U}(k)^{T} \boldsymbol{W} \boldsymbol{U}(k)+2 \boldsymbol{F}^{T}(k) \boldsymbol{U}(k)+C(k),
$$

where

$$
\begin{aligned}
\boldsymbol{W} & =\boldsymbol{\Phi}^{T} \boldsymbol{\Phi}+\sigma \boldsymbol{I}_{3 N \times 3 N}, \\
\boldsymbol{F}(k) & =\boldsymbol{\Phi}^{T} \boldsymbol{\Lambda} \boldsymbol{i}_{\boldsymbol{a} \boldsymbol{b}}(k)-\boldsymbol{\Phi}^{T} \mathcal{I}_{\boldsymbol{a} \boldsymbol{b}}^{\star}(k+1)-\sigma \boldsymbol{U}^{\star}(k),
\end{aligned}
$$

and $C(k)$ is independent of $\boldsymbol{U}(k)$. Furthermore, $\boldsymbol{I}_{3 N \times 3 N}$ is the identity matrix of size $3 N$. The matrices $\boldsymbol{\Phi}$ and $\boldsymbol{\Lambda}$, the current reference sequence $\mathcal{I}_{\boldsymbol{a} \boldsymbol{b}}^{\star}(k+1)$, and the input reference sequence $\boldsymbol{U}^{\star}(k)$ are defined in the appendix. Then, in absence of constraints (i.e., using (19) in $\frac{\partial J_{N}}{\partial \boldsymbol{U}}=0$ ), the unconstrained solution of the problem (16a) is given by:

$$
\boldsymbol{U}_{\mathrm{uc}}(k)=-\boldsymbol{W}^{-1} \boldsymbol{F}(k) \in \mathbb{R}^{3 N},
$$

which does not necessarily belongs to the FCS $\mathbb{U}$ [27].

To obtain the constrained optimal solution $\boldsymbol{U}_{\text {opt }}(k) \in \mathbb{U}$, it is convenient to present the cost function (19) in terms of $\boldsymbol{U}(k)$ and $\boldsymbol{U}_{\mathrm{uc}}(k)$, i.e.:

$$
J_{N}=\left(\boldsymbol{U}(k)-\boldsymbol{U}_{\mathrm{uc}}(k)\right)^{T} \boldsymbol{W}\left(\boldsymbol{U}(k)-\boldsymbol{U}_{\mathrm{uc}}(k)\right)+C(k) .
$$

As per definition, $\boldsymbol{W}$ is a symmetric and positive definite matrix for $\sigma>0$, see (20a). Then, to reformulate (16a) as an ILS-problem, a unique invertible lower triangular matrix $\boldsymbol{H} \in \mathbb{R}^{3 N \times 3 N}$ is obtained by performing the Cholesky decomposition [37], [38] to $\boldsymbol{W}^{-1}$, i.e.:

$$
\boldsymbol{W}^{-1}=\boldsymbol{H}^{-1} \boldsymbol{H}^{-T} .
$$

Hence, $\boldsymbol{H}$ satisfies the following expression:

$$
\boldsymbol{W}=\boldsymbol{H}^{T} \boldsymbol{H} .
$$

Now, the cost function (22) can be rewritten in terms of $\boldsymbol{H}$ as:

$$
J_{N}=\|\boldsymbol{H} \boldsymbol{U}(k)-\mathcal{Y}(k)\|_{2}^{2}+C(k)
$$

where

$$
\mathcal{Y}(k)=\boldsymbol{H} \boldsymbol{U}_{\mathrm{uc}}(k) .
$$

Finally, by using (25) the optimization problem (16a) becomes:

$$
\boldsymbol{U}_{\text {opt }}(k)=\arg \left\{\min _{\boldsymbol{U}(k)}\|\boldsymbol{H} \boldsymbol{U}(k)-\mathcal{Y}(k)\|_{2}^{2}\right\},
$$


which is subject to the constraints (16b)-(16d). Notice that (27) is a quantization problem of ILS form. It is also important to highlight that obtaining the optimal solution $\boldsymbol{U}_{\text {opt }}(k)$ by performing the quantization as per (27) is equivalent to solve the minimization in (16a).

\section{B. The SDA as an optimizer}

The SDA is based on branch and bound technique that can reduce the computational effort compared to the ESA, see [23], [35]. In general, it works by defining a sphere of radius $d(k)>$ 0 and center $\mathcal{Y}(k)$ in (27). The computational effort required to obtain the optimal solution $\boldsymbol{U}_{\text {opt }}(k)$ largely depends on the size of this sphere (i.e., $d(k)$ ). The value of $d(k)$ should be large enough, so the sphere contains at least one tentative solution. In this work, the initial value of $d(k)$ is calculated by the following deterministic method:

$$
d(k)=\left\|\boldsymbol{H} \boldsymbol{U}_{\text {be }}(k)-\mathcal{Y}(k)\right\|_{2}^{2},
$$

where $\boldsymbol{U}_{\mathrm{be}}(k)=\left\lfloor\boldsymbol{U}_{\mathrm{uc}}(k)\right\rceil \in \mathbb{U}$ is found by applying the Babai estimation [39]. It is worth noting that $\boldsymbol{U}_{\text {be }}(k)$ may not be the optimal solution but feasible to initialize the algorithm.

Considering the initial radius, the SDA starts searching tentative solutions $\boldsymbol{U}(k)$ in a repetitive manner by satisfying the following condition:

$$
\|\boldsymbol{H} \boldsymbol{U}(k)-\mathcal{Y}(k)\|_{2}^{2} \leqslant d(k) .
$$

This is the condition for $\boldsymbol{U}(k)$ belong to the sphere of radius $d(k)$ and center $\mathcal{Y}(k)$. The computational benefit involved in this repetitive searching method comes from the fact of using $\boldsymbol{H}$ in (27), which can be seen by extending (29) as follows:

$$
\left(h_{11} U_{1}-\mathcal{Y}_{1}\right)^{2}+\left(h_{21} U_{1}+h_{22} U_{2}-\mathcal{Y}_{2}\right)^{2}+\cdots \leqslant d^{2}(k),
$$

where $h_{i j}$ is the $(i, j)^{t h}$-element of $\boldsymbol{H} ; U_{i}$ and $\mathcal{Y}_{i}$ refer to the $i^{\text {th }}$-element of $\boldsymbol{U}(k)$ and $\mathcal{Y}(k)$, respectively. At the left-hand side of (30), the first term depends on $U_{1}$, the second term on $\left\{U_{2}, U_{1}\right\}$ and so on. This facilitates to sequentially perform an element-wise computation.

A flow diagram of the SDA for three-phase $\eta$-cell CHB inverter is shown in Fig. 3. Here, $i$ denotes the entry index of a vector or matrix, where $i \in\{1, \ldots, 3 N\}$, and $c_{o}$ used to set the $i^{t h}$ entry of $\boldsymbol{U}(k)$, where $c_{o} \in\{0, \ldots, 2 \eta\}$. Furthermore, $\boldsymbol{\rho}$ is a $(3 N+1)$-dimensional vector which is initialized as zerovector. As per (30), the algorithm starts forming a tentative solution $\boldsymbol{U}(k)$ element by element (i.e., from $U_{1}$ down to $U_{3 N}$ ), where each element $U_{i}$ belongs to the phase voltage level set $\mathbb{V}$ and satisfies (16d). Here, the constraint (16d) is checked by using the optimal solution $\boldsymbol{U}_{\text {opt }}(k-1)$ found at the previous instant. Note that each entry of $\boldsymbol{U}(k)$ starts from $-\eta$ and ends at $+\eta$ with an incremental change of $c_{o}$. For an element $U_{i}$, if the squared distance $\rho_{i}$ violates the condition in (30), it implies that all associated computations for the consecutive elements (i.e., $U_{i+1}$ to $U_{3 N}$ ) will provide an even larger value of $\rho_{i}$. Therefore, the algorithm does not explore these elements avoiding, thus, unnecessary calculations. On the other hand, if the $\rho_{i}$ for any $U_{i}$ is smaller than the present value of $d^{2}$, then it computes for the next element $U_{(i+1)}$. Proceeding in this manner, whenever $\boldsymbol{U}(k)$ is fully formed,

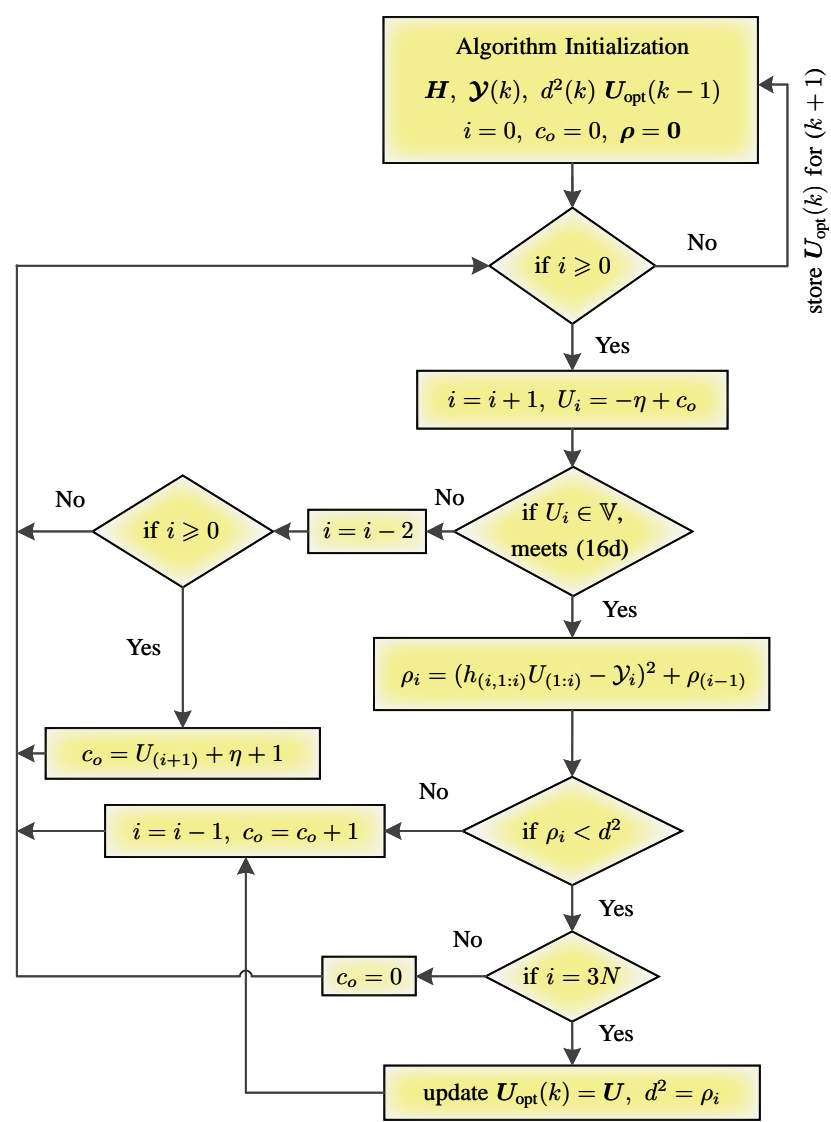

Fig. 3. Flow diagram of the SDA for a three-phase $\eta$-cell CHB inverter.

i.e., $i=3 N$, it implies that a tentative solution $\boldsymbol{U}(k)$ is found. Then, it is updated as $\boldsymbol{U}_{\text {opt }}$ and, so the associated $\rho_{i}$ as $d^{2}$. At every update stage, the sphere size is reduced since the updated radius $d$ is smaller than its predecessor. This helps to keep the tentative solution set to be explored as small as possible, which ensures less computational effort [23]. The optimization process terminates when the sphere is reduced to the point where only one tentative solution is contained in it, which is, in fact, the optimal solution $\boldsymbol{U}_{\mathrm{opt}}(k)$ in (27).

\section{ANALYSIS OF THE PROPOSED FCS-MPC FORMULATION}

In this section, the role of the weighting factor $\sigma$ on the proposed FCS-MPC strategy is analyzed in a three-dimensional (3-D) space taking into consideration the quantization problem (27). This analysis is key to understand how the proposed MPC formulation can handle both control targets (current references tracking and CMV minimization) in only one optimization problem. Notice that, a horizon length $N>1$ produces a dimension of the FCS $\mathbb{U}$ greater than 3 , as stated by $\mathbb{U}=$ $\mathcal{U}^{N}=\mathbb{V}^{3 N}$ in (15), in which case only a mathematical but no graphical representation is feasible. Therefore, to clearly present the problem and have a 3-D representation of (27), $N=1$ is chosen with $\mathbb{U}=\mathcal{U}=\mathbb{V}^{3}$. In this case, the cost function (13) becomes:

$$
J_{1}=\left\|\boldsymbol{i}_{\boldsymbol{a} \boldsymbol{b}}(k+1)-\boldsymbol{i}_{\boldsymbol{a} \boldsymbol{b}}^{\star}(k+1)\right\|_{2}^{2}+\sigma\left\|\boldsymbol{u}(k)-\boldsymbol{u}^{\star}(k)\right\|_{2}^{2} .
$$




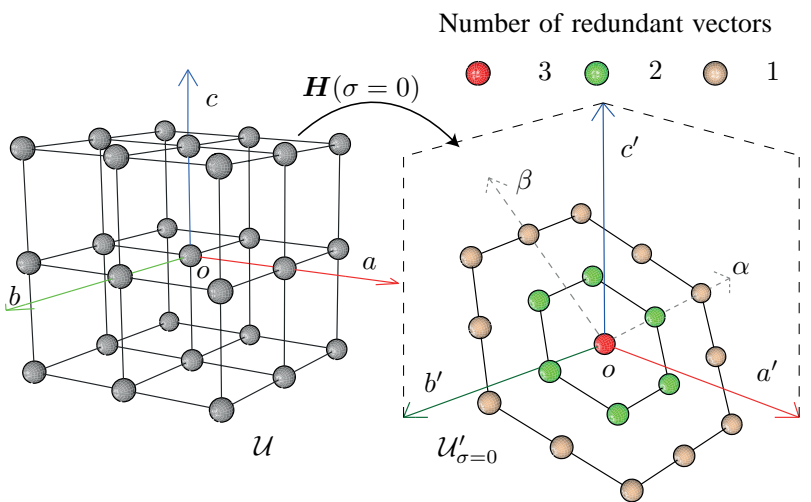

(a)

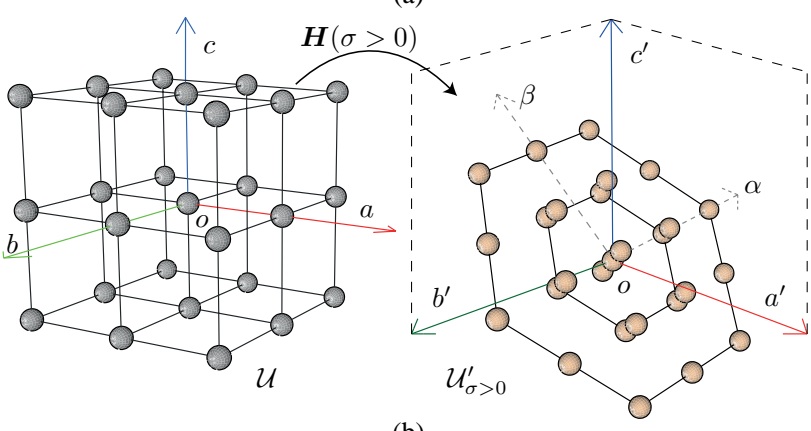

(b)

Fig. 4. Linear transformation of control inputs by the matrix $\boldsymbol{H}$ for a threephase one-cell ( $\eta=1$ ) CHB converter when: (a) $\sigma=0$; and (b) $\sigma>0$.

Moreover, to have a comparison with the basic single-step FCS-MPC, this analysis examines the same three-phase onecell $(\eta=1)$ CHB converter discussed in Section II-B as an example. Hence, the control inputs belong to the cubelattice in $a b c$-framework (i.e., the FCS $\mathcal{U}$, see Figs. 2 and 4). The OCI is one of the lattice points which minimizes the cost function (31). As can be noticed in (27), the matrix $\boldsymbol{H}$ is a linear transformation over the control inputs, which yields a transformed FCS $\mathcal{U}^{\prime}$ in the new $a^{\prime} b^{\prime} c^{\prime}$-framework, i.e., $\mathcal{U}^{\prime}=\boldsymbol{H} \cdot \mathcal{U}$. Therefore, the OCI can be obtained from $\mathcal{U}^{\prime}$ by performing the optimization (27) in the $a^{\prime} b^{\prime} c^{\prime}$-framework. Note that the properties of $\boldsymbol{H}$ depend on $\boldsymbol{W}$ in (20a), in particular on the weighting factor $\sigma$. For $N=1$ case, $\boldsymbol{W}$ becomes:

$$
\boldsymbol{W}=\boldsymbol{B}^{T} \boldsymbol{B}+\sigma \boldsymbol{I}_{3 \times 3}
$$

When $\sigma=0, J_{1}$ in (31) becomes the basic single-step cost function (11), which only considers the current references tracking. In this case, $\boldsymbol{W}=\boldsymbol{B}^{T} \boldsymbol{B}$, which is singular. Hence, $\boldsymbol{H} \in \mathbb{R}^{3 \times 3}$ is also singular, i.e., the sum of elements in each row is zero:

$$
\sum_{j=1}^{3} h_{i j}=0 ; \quad i \in\{1,2,3\},
$$

where $h_{i j}$ is the $(i, j)^{t h}$-entries of $\boldsymbol{H}$. Now, the linear transformation $\boldsymbol{H}$ over the cube-lattice $\mathcal{U}$ yields a hexagon $\mathcal{U}_{\sigma=0}^{\prime}$ in the $a^{\prime} b^{\prime} c^{\prime}$-framework as depicted in Fig. 4a. Notice that this hexagon resembles the standard hexagon in the $\alpha \beta$ framework having the same redundant vectors, see Figs. 2 and $4 \mathrm{a}$. Here, the redundant vectors at the center are obtained when all elements of the input vector $\boldsymbol{u}(k) \in \mathcal{U}$ are equal (i.e., $\left.v_{\ell a}=v_{\ell b}=v_{\ell c}\right)$. For the elements of $\boldsymbol{u}(k) \in \mathcal{U}$ that satisfy the following:

$$
\begin{gathered}
v_{\ell p}=v_{\ell q} ; \quad v_{\ell r}=v_{\ell p} \pm 1 ; \\
p \neq q \neq r ; \quad p, q, r \in\{a, b, c\},
\end{gathered}
$$

form the redundant vectors located at six vertices of the inner layer of the hexagon. The remaining vectors positioned at the outer layer of the hexagon are distinct, i.e., no redundancies. Thereby, the redundant vectors located in the same position give the same output current but might have a different CMV. Thus, for the case when $\sigma=0$, there may exist more than one input that gives the same minimum cost value. Therefore, the OCI may result in balanced sinusoidal current with high (uncontrolled) CMV.

For the case when $\sigma>0$, the cost function (31) contains the desired CMV information through the control input reference $\boldsymbol{u}^{\star}(k)$ which is designed in (14). Moreover, matrices $\boldsymbol{W}$ and $\boldsymbol{H}$ are now non-singular. Therefore, all transformed vectors under $\mathcal{U}_{\sigma>0}^{\prime}$ in the $a^{\prime} b^{\prime} c^{\prime}$-framework are distinct in terms of cost value, see Fig. 4b. Notice that the redundant vectors located in the same position when $\sigma=0$ (Fig. 4a), are pulled apart as $\sigma$ is increased ( $\sigma>0$, Fig. 4b). Hence, when $\sigma>0$, there exist only one OCI that gives the minimum current tracking error and reduced CMV. Consequently, by solving only one optimization problem the system can achieve threephase balanced current with reduced CMV. It is important to note that the lattice structure changes for different values of $\sigma$. However, the performance of the SDA is affected only for large values of $\sigma$, which are not considered in this work.

The analysis presented in this section can be extended for converters with a larger number of cells $(\eta>1)$ and long prediction horizons $(N>1)$. For $\eta>1$ when $N=1$, the size of the hexagon in the $a^{\prime} b^{\prime} c^{\prime}$-framework increases by incorporating more layers with redundant vectors. On the contrary, for $N>1$ when $\eta \geq 1$, the hexagon-shape can not be guaranteed. However, the mathematical analysis presented here still holds.

\section{Case Study: Two-Cell CHB Inverter}

This section highlights the effectiveness of the proposed MPC formulation, when it is used to govern a three-phase two-cell $(\eta=2)$ CHB inverter with passive RL loads as an illustrative example. The main system parameters are shown in Table I.

Table I

SYSTEM PARAMETERS

\begin{tabular}{ccc}
\hline \hline Variable & Description & Values \\
\hline$P_{r}$ & Rated three-phase power & $3.45 \mathrm{~kW}$ \\
$V_{d c}$ & dc voltage supply per HB & $180 \mathrm{~V}$ \\
$I^{\star}$ & Current reference amplitude & $7 \mathrm{~A}$ \\
$f$ & Load current frequency & $50 \mathrm{~Hz}$ \\
$R$ & Load resistor & $47 \Omega$ \\
$L$ & Load inductor & $15 \mathrm{mH}$ \\
$f_{s}$ & Sampling frequency & $10 \mathrm{kHz}$ \\
\hline \hline
\end{tabular}


(a)

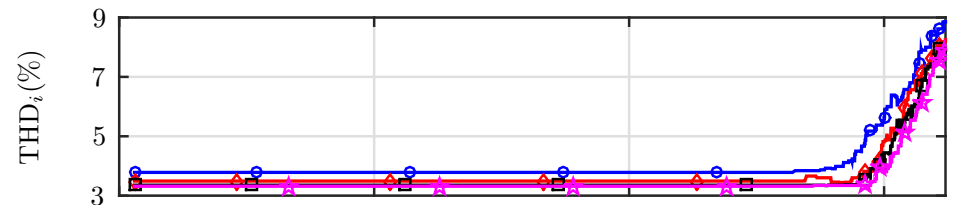

(c)

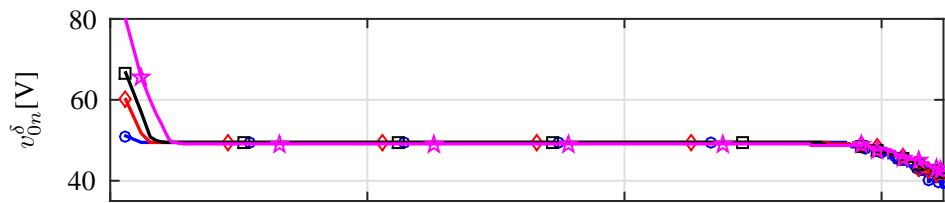

(e)

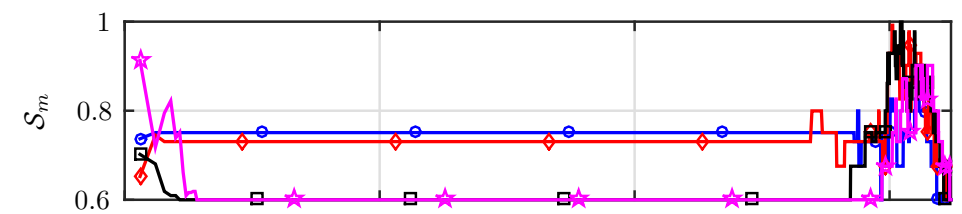

(g)

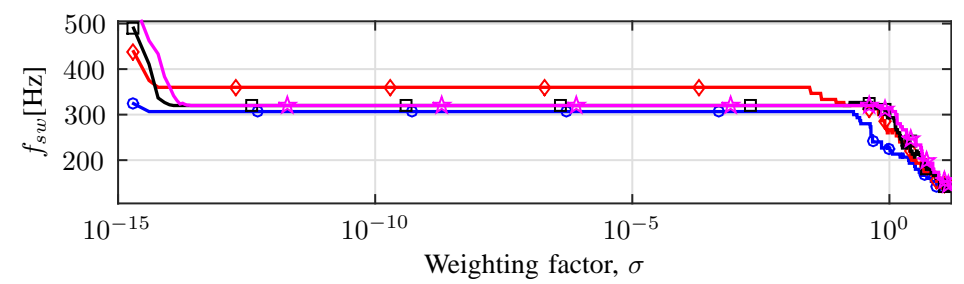

(b)

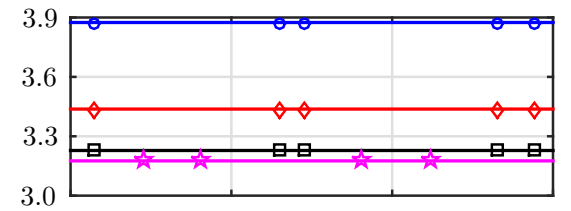

(d)

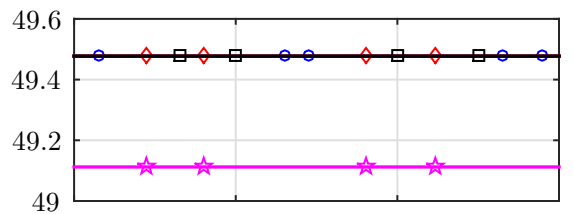

(f)

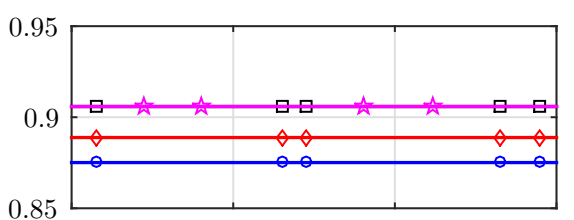

(h)

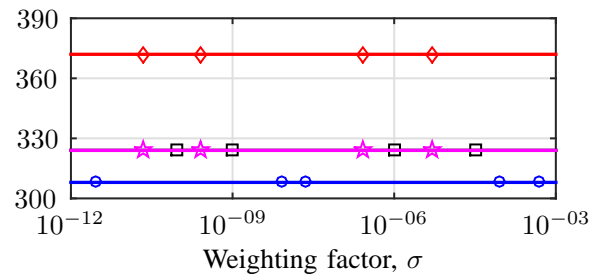

Fig. 5. System performance analysis of the proposed FCS-MPC strategy by considering the system parameters shown in Table. I. THD of load currents $\left(\operatorname{THD}_{i}\right)$, standard deviation of CMV $\left(v_{0 n}^{\delta}\right)$, inverter voltage symmetry $\left(\mathcal{S}_{m}\right)$ and average devise switching frequency $\left(f_{s w}\right)$ are presented as a function of $\sigma$ in semilogarithmic plots for $N \in\{1,2,3,10\}$. Color notations: $-\bigcirc-O-(N=1) ;-\diamond-\diamond-(N=2) ;-\square-\square-(N=3)$; and $-\star-\star-(N=10)$. Figures (b), (d), (f) and (h) are the close-up views of their corresponding plots (a), (c), (e) and (g), respectively for $\sigma \in\left[10^{-12}, 10^{-3}\right]$.

\section{A. Controller Design}

To achieve a desired system performance, the controller settings (horizon length $N$ and weighting factor $\sigma$ ) need to be tuned. At first, the proposed strategy is simulated for $N=1,2,3$ and 10 . For each value of $N$, the simulation is performed for different values of $\sigma$ ranging from $10^{-15}$ to 16 . Then, the steady-state performance is investigated in terms of: the load currents THD $\left(\mathrm{THD}_{i}\right)$, the standard deviation of CMV $\left(v_{0 n}^{\delta}\right)$, the inverter voltage symmetry $\left(\mathcal{S}_{m}\right)$ and the average device switching frequency $\left(f_{s w}\right)$. Here, $v_{0 n}^{\delta}$ defines how the values of CMV over a period are spread out from their average value. The term $\mathcal{S}_{m}$ refers to the identical switching pattern in an inverter voltage $v_{y n}$, in terms of the quarter-wave symmetry. This is particularly important for grid-connected systems, where even harmonics injection must be strictly limited to small values, see e.g. IEEE-Std 519, where the even harmonics are limited to $25 \%$ of the odd harmonics. In this work, $\mathcal{S}_{m}$ is quantified by the correlations of the four quarter-waveforms, $\mathcal{Q}_{1}\left(v_{y n}\right) \ldots \mathcal{Q}_{4}\left(v_{y n}\right)$, over one fundamental period. This is obtained by using a well-known statistical analysis tool named Pearson's correlation-coefficient [40], $\mathcal{P} \in[0,1]$, by computing the following expression:

$$
\mathcal{S}_{m}=\frac{1}{3} \sum_{y=a}^{c} \frac{1}{2}\left(S_{v_{y n}}^{+}+S_{v_{y n}}^{-}\right),
$$

where $S_{v_{y n}}^{+}=\mathcal{P}\left(\mathcal{Q}_{1}\left(v_{y n}\right), \mathcal{Q}_{2}^{\prime}\left(v_{y n}\right)\right), \quad S_{v_{y n}}^{-}=$ $\mathcal{P}\left(\mathcal{Q}_{3}\left(v_{y n}\right), \mathcal{Q}_{4}^{\prime}\left(v_{y n}\right)\right)$, in which $\mathcal{Q}_{2,4}^{\prime}\left(v_{y n}\right)$ is the mirrored version of $\mathcal{Q}_{2,4}\left(v_{y n}\right)$. Hence, higher the value of $\mathcal{S}_{m}$ (maximum of 1 ), the more symmetric the inverter voltages are and lower the rate of even harmonics injection to the system. Considering the four aforementioned metrics, the simulation results are presented in semilogarithmic plots as a function of $\sigma$ for $N=1,2,3$ and 10 in Fig. 5.

1) Weighting factor $(\sigma)$ tuning: From Fig. 5 , it can be seen that the results are not sensitive to the range $\sigma \in$ $\left[10^{-12}, 10^{-3}\right]$. When $\sigma \approx 10^{0}$ or larger, $v_{0 n}^{\delta}$ and $f_{s w}$ are reduced. However, the $\mathrm{THD}_{i}$ and $\mathcal{S}_{m}$ are increased. Generally, higher values of $\sigma$ reduces the dynamic performance of the FCS-MPC, see [31], where $\sigma$ is represented as $R$. Hence, to achieve a desired closed-loop performance of the system with fast dynamic, smaller values of $\sigma$ are selected. Based on this analysis, $\sigma=10^{-6}$ is chosen from the non-sensitive range (i.e., $\sigma \in\left[10^{-12}, 10^{-3}\right]$ ).

2) Prediction horizon $(N)$ tuning: It can be observed from the close-up view of Fig. 5 that $\mathrm{THD}_{i}, v_{0 n}^{\delta}$ and $\mathcal{S}_{m}$ are gradually reduced as the horizon length is increased. For $N=3$, there is $17.05 \%$ reduction in the $\mathrm{THD}_{i}$ compared to the case when $N=1$. On the contrary, moving to $N=10$ results in $18.61 \%$ THD reduction, which means there is only a $1.56 \%$ improvement over $N=3$, see Fig. 5 b. According to Fig. 5d and Fig. 5f, there are no considerable improvements in terms of $v_{0 n}^{\delta}$ and $\mathcal{S}_{m}$ at $N>1$. Regarding $f_{s w}$, it can be seen from Fig. 5h that $f_{s w}$ has shown irregular variations. For $N=3$ and 10 , the value of $f_{s w}$ is $326 \mathrm{~Hz}$, whereas at $N=1$ it is $312 \mathrm{~Hz}$. From the above analysis, it is clear that $N=10$ case offers negligible improvement in the system performance compared to $N=3$. Moreover, it requires enormous computations, which is intractable for real-time implementation. Consequently, $N=3$ is chosen considering the trade-off between the system performance and the computational burden. 
(a)

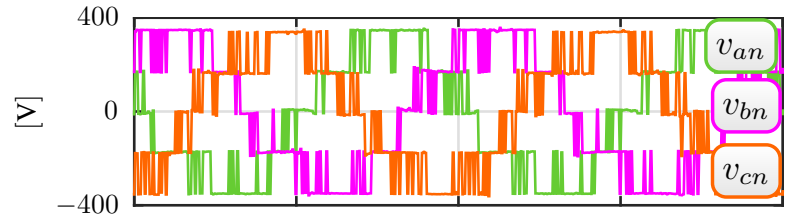

(b)

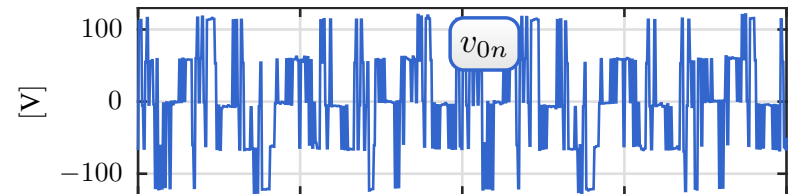

(c)

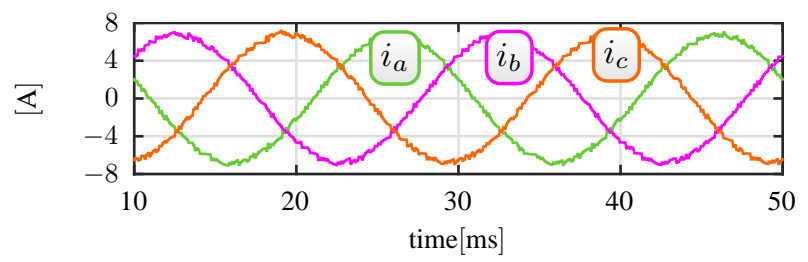

Fig. 6. Steady-state performance for a basic single-step FCS-MPC $(N=$ $1, \sigma=0$ ): (a) inverter voltages; (b) CMV; (c) load currents. (a)

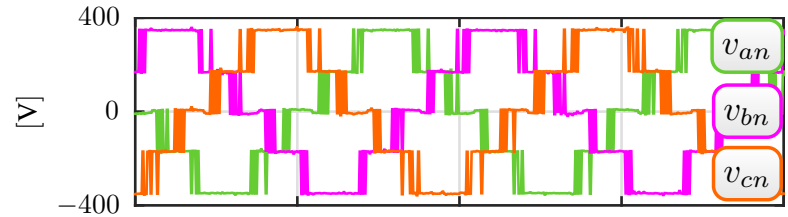

(b)

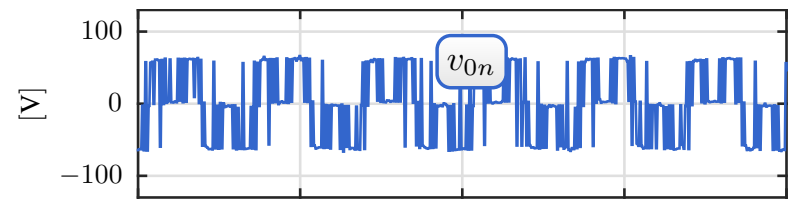

(c)

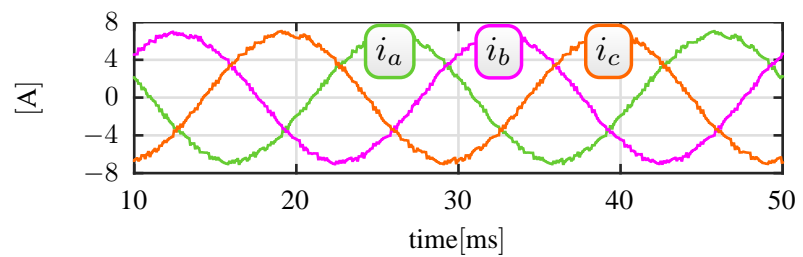

Fig. 7. Steady-state performance for the proposed FCS-MPC with single-step prediction horizon $\left(N=1, \sigma=10^{-6}\right)$ : (a) inverter voltages; (b) $\mathrm{CMV}$; (c) load currents.

\section{B. Experimental Results}

The experimental validation of the proposed FCS-MPC formulation was performed in a dSPACE DS1006 system. Here, the state of the power switches was implemented in an FPGA module DS5203. The controller considers the wellknown time delay compensation [41] in the formulation.

1) Comparison with basic single-step FCS-MPC: At first, the CHB inverter is governed with the basic single-step FCSMPC $(N=1, \sigma=0)$, which tracks only the output current references. Here, the standard ESA is used as an optimizer. The results presented in Fig. 6 and Table II show that the proposed FCS-MPC with single-step prediction horizon $\left(N=1, \sigma=10^{-6}\right)$ as depicted in Fig. 7, provides a steadystate performance with a $54.4 \%$ improvement in the symmetry, and a reduction of $24.8 \%$ in the CMV standard deviation and $14.5 \%$ in the average switching frequency. As anticipated, the THD for the currents shown in Fig. 7c remains almost similar, i.e., $\mathrm{THD}_{i}=4.03 \%$. Notice that the SDA significantly reduces the execution time, $T_{e}$ (i.e., 3.6 times faster) compared with (a)

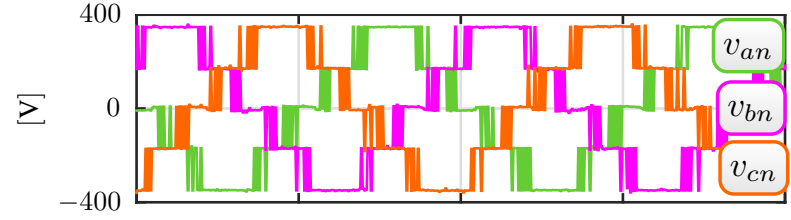

(b)

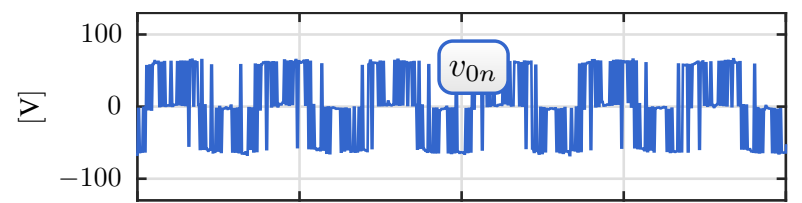

(c)

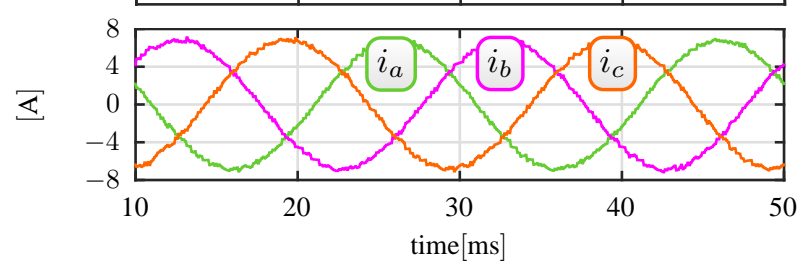

Fig. 8. Steady-state performance for the proposed FCS-MPC with long prediction horizon $\left(N=3, \sigma=10^{-6}\right)$ : (a) inverter voltages; (b) CMV; (c) load currents.

Table II

SteAdy State PERformanCES: EXPERIMENTAL Results

\begin{tabular}{lccc}
\hline \hline Horizon length & $N=1$ & $N=1$ & $N=3$ \\
Weighting factor & $\sigma=0$ & $\sigma=10^{-6}$ & $\sigma=10^{-6}$ \\
Optimizer & ESA & SDA & SDA \\
\hline Execution Time, $T_{e}\left(\% \text { of } T_{s}\right)^{\text {a }}$ & $39.8 \%$ & $11.1 \%$ & $92.2 \%$ \\
THD of the load currents, THD $_{i}$ & $4.01 \%$ & $4.03 \%$ & $3.36 \%$ \\
Standard deviation of CMV, $v_{0 n}^{\delta}$ & $64.5 \mathrm{~V}$ & $48.51 \mathrm{~V}$ & $47.7 \mathrm{~V}$ \\
Inverter voltage symmetry, $\mathcal{S}_{m}$ & 0.57 & 0.88 & 0.92 \\
Avg. device switching freq., $f_{s w}$ & $387.5 \mathrm{~Hz}$ & $331.25 \mathrm{~Hz}$ & $334.12 \mathrm{~Hz}$
\end{tabular}

${ }^{\mathrm{a}}$ : Sampling period $T_{s}=100 \mu \mathrm{s}$.

the ESA. This allows one to implement the proposed FCSMPC with longer prediction horizons.

2) Long prediction horizon performance: To explore the benefits of long prediction horizon implementation, the proposed FCS-MPC is carried out with the controller settings ( $N=3, \sigma=10^{-6}$ ) obtained in Section VI-A. The results are presented in Fig. 8 and Table II. For $N>1$, the execution time $T_{e}$ exceeds the time limit imposed by $T_{s}$ when using ESA. However, the SDA implementation still ensures $T_{e}$ to be within $T_{s}$ for $N=3$. This test shows that the average device switching frequency and CMV do not present noticeable changes. Nevertheless, the system experiences an improvement of $16.6 \%$ in $\mathrm{THD}_{i}$ with an inverter voltage symmetry $\mathcal{S}_{m}=0.92$, which means the inverter voltages are near symmetric.

3) Dynamic performance: The dynamic behavior of the proposed strategy for a step change in the amplitude of current references is depicted in Fig. 9. The results are shown for phase $a$ only. When the step change in the current references (from $-3.5 \mathrm{~A}$ to $7 \mathrm{~A}$ ) is applied at $t=20 \mathrm{~ms}$, the load current 
(a)

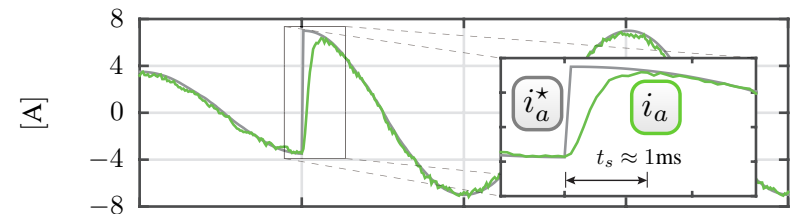

(b)

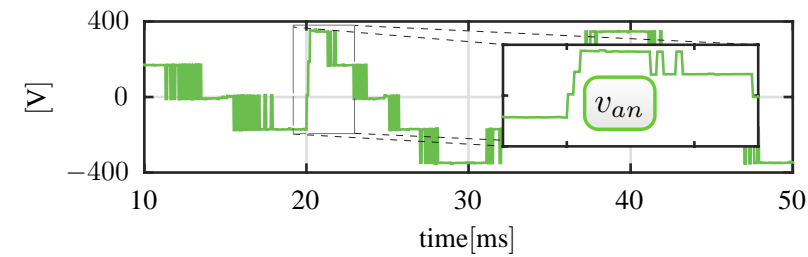

Fig. 9. Dynamic performance under step change in the current reference for the proposed FCS-MPC with long prediction horizon $\left(N=3, \sigma=10^{-6}\right)$ (a) load current; and (b) inverter voltage. Results are shown for phase $a$ only. (a)

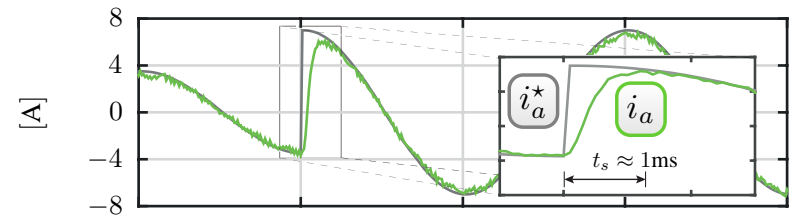

(b)

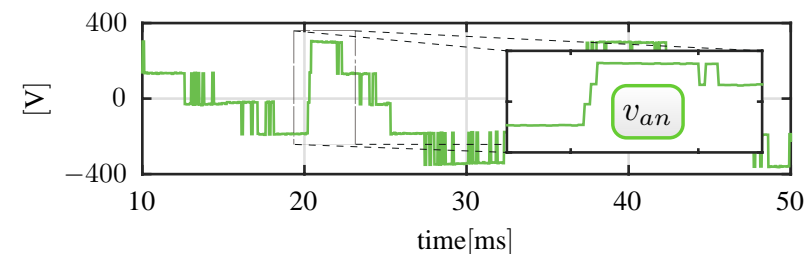

Fig. 10. Dynamic performance under step change in the current reference for a basic single-step FCS-MPC ( $N=1, \sigma=0)$ : (a) load current; and (b) inverter voltage. Results are shown for phase $a$ only.

quickly reaches its desired reference with an approximate settling time, $t_{s}=1 \mathrm{~ms}$. As can be seen from the close-up view, the inverter voltage changes are limited to one step. This is due to the voltage level constraint (16d) in the optimization problem formulation which ensures the lower $d v / d t$ ratings at the load. For a fair comparison, the basic single-step FCSMPC also considered (16d), and the dynamic performance is carried out for the same test condition, see Fig. 10. This result proves that the proposed strategy does not affect the inherent fast dynamic performance of the basic single-step FCS-MPC even for long prediction horizon implementations.

\section{CONCLUSION}

A suitable long prediction horizon MPC formulation for $\mathrm{CHB}$ inverters has been proposed. The MPC was formulated to include the full steady-state system information in terms of output current and output voltage references in the original stationary $a b c$-framework. Since the CMV information is preserved in the $a b c$-framework, the control input references have been designed to obtain a minimum CMV. Moreover, to deal with the computational burden introduced by a long prediction horizon implementation, the proposed MPC formulation was transformed into an equivalent optimization problem that was effectively solved by the SDA. Based on this transformation, a graphical analysis of the proposed MPC formulation has been presented. Particularly, it has been shown that the weighting factor plays an important role to obtain a unique optimal solution which demonstrates that current references tracking and CMV minimization can be achieved in a single optimization problem even for long prediction horizon. Experimental results have shown that for the single-step case, the proposed FCS-MPC outperforms the basic single-step FCS-MPC in terms of inverter voltages symmetry, average device switching frequency, and CMV while reducing the required execution time. Besides these benefits, results for the long prediction horizon implementation have shown a significant improvement in the current THD without affecting the inherent fast dynamic performance of the basic single-step FCS-MPC.

\section{APPENDIX}

The matrices and vectors used in (20) are given as:

$$
\begin{gathered}
\boldsymbol{\Phi}=\left[\begin{array}{ccccc}
\boldsymbol{B} & 0 & \ldots & 0 & 0 \\
\boldsymbol{A} \boldsymbol{B} & \boldsymbol{B} & \ldots & 0 & 0 \\
\vdots & \vdots & \ddots & \vdots & \vdots \\
\boldsymbol{A}^{N-1} \boldsymbol{B} & \boldsymbol{A}^{N-2} \boldsymbol{B} & \ldots & \boldsymbol{A} \boldsymbol{B} & \boldsymbol{B}
\end{array}\right], \quad \boldsymbol{\Lambda}=\left[\begin{array}{c}
\boldsymbol{A} \\
\boldsymbol{A}^{2} \\
\vdots \\
\boldsymbol{A}^{N}
\end{array}\right], \\
\mathcal{I}_{\boldsymbol{a} \boldsymbol{b}}^{\star}(k)=\left[\begin{array}{c}
\boldsymbol{i}_{\boldsymbol{a} \boldsymbol{b}}^{\star}(k+1) \\
\boldsymbol{i}_{\boldsymbol{a} \boldsymbol{b}}^{\star}(k+2) \\
\vdots \\
\boldsymbol{i}_{\boldsymbol{a} \boldsymbol{b}}^{\star}(k+N)
\end{array}\right], \boldsymbol{U}^{\star}(k)=\left[\begin{array}{c}
\boldsymbol{u}^{\star}(k) \\
\boldsymbol{u}^{\star}(k+1) \\
\vdots \\
\boldsymbol{u}^{\star}(k+N-1)
\end{array}\right] .
\end{gathered}
$$

\section{REFERENCES}

[1] S. Kouro, M. Malinowski, K. Gopakumar, J. Pou, L. Franquelo, B. Wu, J. Rodríguez, M. A. Pérez, and J. Leon, "Recent Advances and Industrial Applications of Multilevel Converters," IEEE Trans. Ind. Electron., vol. 57, no. 8, pp. 2553-2580, 2010.

[2] T. A. Meynard, H. Foch, P. Thomas, J. Courault, R. Jakob, and M. Nahrstaedt, "Multicell converters: basic concepts and industry applications," IEEE Trans. Ind. Electron., vol. 49, no. 5, pp. 955-964, 2002.

[3] J. Rodriguez, S. Bernet, P. K. Steimer, and I. E. Lizama, "A Survey on Neutral-Point-Clamped Inverters," IEEE Trans. Ind. Electron., vol. 57, no. 7, pp. 2219-2230, 2010.

[4] M. Malinowski, K. Gopakumar, J. Rodríguez, and M. A. Pérez, "A Survey on Cascaded Multilevel Inverters," IEEE Trans. Ind. Electron., vol. 57, no. 7, pp. 2197-2206, 2010.

[5] J. I. Leon, K. Kouro, L. G. Franquelo, J. Rodriguez, and B. Wu, "The Essential Role and the Continuous Evolution of Modulation Techniques for Voltage-Source Inverters in the Past, Present, and Future Power Electronics," IEEE Trans. Ind. Electron., vol. 63, no. 5, pp. 2688-2701, 2016.

[6] S. Vazquez, J. I. Leon, L. G. Franquelo, J. Rodriguez, H. A. Young, A. Marquez, and P. Zanchetta, "Model Predictive Control: A Review of Its Applications in Power Electronics," IEEE Ind. Electron. Mag., vol. 8, no. 1, pp. 16-31, March 2014.

[7] J. Rodríguez, M. P. Kazmierkowski, J. R. Espinoza, P. Zanchetta, H. Abu-Rub, H. A. Young, and C. A. Rojas, "State of the Art of Finite Control Set Model Predictive Control in Power Electronics," IEEE Trans. Ind. Informat., vol. 9, no. 2, pp. 1003-1016, May 2013.

[8] P. Acuna, R. P. Aguilera, A. M. Y. M. Ghias, M. Rivera, C. R. Baier, and V. G. Agelidis, "Cascade-Free Model Predictive Control for SinglePhase Grid-Connected Power Converters," IEEE Trans. Ind. Electron., vol. 64, no. 1, pp. 285-294, Jan. 2017.

[9] S. Vazquez, A. Marquez, R. P. Aguilera, D. Quevedo, J. I. Leon, and L. G. Franquelo, "Predictive Optimal Switching Sequence Direct Power Control for Grid-Connected Power Converters," IEEE Trans. Ind. Electron., vol. 62, no. 4, pp. 2010-2020, 2015.

[10] R. P. Aguilera, P. Lezana, and D. E. Quevedo, "Switched Model Predictive Control for Improved Transient and Steady-State Performance," IEEE Trans. Ind. Informat., vol. 11, no. 4, pp. 968-977, Aug. 2015. 
[11] P. Acuna, L. Moran, M. Rivera, R. P. Aguilera, R. Burgos, and V. G. Agelidis, "A Single-Objective Predictive Control Method for a Multivariable Single-Phase Three-Level NPC Converter-Based Active Power Filter," IEEE Trans. Ind. Electron., vol. 62, no. 7, pp. 4598-4607, Jul. 2015.

[12] R. P. Aguilera, P. Lezana, and D. E. Quevedo, "Finite-Control-Set Model Predictive Control With Improved Steady-State Performance," IEEE Trans. Ind. Informat., vol. 9, no. 2, pp. 658-667, May 2013.

[13] D. E. Quevedo, R. P. Aguilera, M. A. Pérez, P. Cortés, and R. Lizana, "Model Predictive Control of an AFE Rectifier With Dynamic References," IEEE Trans. Power Electron., vol. 27, no. 7, pp. 3128-3136, 2012.

[14] R. P. Aguilera, P. Acuna, P. Lezana, G. Konstantinou, B. Wu, S. Bernet, and V. G. Agelidis, "Selective Harmonic Elimination Model Predictive Control for Multilevel Power Converters," IEEE Trans. Power Electron., vol. 32, no. 3, pp. 2416-2426, Mar. 2017.

[15] P. Cortés, A. Wilson, S. Kouro, J. Rodríguez, and Abu-Rub, "Model Predictive Control of Multilevel Cascaded H-Bridge Inverters," IEEE Trans. Ind. Electron., vol. 57, no. 8, pp. 2691-2699, 2010.

[16] R. P. Aguilera, P. Acuna, Y. Yu, G. Konstantinou, C. D. Townsend, B. Wu, and V. G. Agelidis, "Predictive Control of Cascaded H-Bridge Converters Under Unbalanced Power Generation," IEEE Trans. Ind. Electron., vol. 64, no. 1, pp. 4-13, Jan. 2017.

[17] A. Hu, D. Xu, B. Wu, J. Wang, and J. Su, "Reference-TrajectoryOptimized SVM for High-Power Current-Source Converters to Improve Harmonic Performance and Reduce Common-Mode Voltage," IEEE Trans. Power Electron., vol. 30, no. 7, pp. 3488-3498, July 2015.

[18] P. Li, G. P. Adam, Y. Hu, D. Holliday, and B. W. Williams, "ThreePhase AC-Side Voltage-Doubling High Power Density Voltage Source Converter With Intrinsic Buck-Boost Cell and Common-Mode Voltage Suppression," IEEE Trans. Power Electron., vol. 30, no. 9, pp. 52845298, Sept 2015.

[19] H. Gao, B. Wu, D. Xu, M. Pande, and R. P. Aguilera, "Common-Mode Voltage Reduced Model Predictive Control Scheme for Current Source Converter-Fed Induction Motor Drives," IEEE Trans. Power Electron., vol. PP, no. 99, pp. 1-1, 2016.

[20] C. Xia, T. Liu, T. Shi, and Z. Song, "A simplified finite-controlset model-predictive control for power converters," IEEE Trans. Ind. Informat., vol. 10, no. 2, pp. 991-1002, 2014.

[21] R. Vargas, U. Ammann, J. Rodriguez, and J. Pontt, "Predictive strategy to reduce common-mode voltages on power converters," in Proc. IEEE PESC, June 2008, pp. 3401-3406.

[22] P. Karamanakos, T. Geyer, N. Oikonomou, F. D. Kieferndorf, and S. Manias, "Direct Model Predictive Control: A Review of Strategies That Achieve Long Prediction Intervals for Power Electronics," IEEE Ind. Electron. Mag., vol. 8, no. 1, pp. 32-43, March 2014.

[23] T. Geyer and D. E. Quevedo, "Multistep Finite Control Set Model Predictive Control for Power Electronics," IEEE Trans. Power Electron., vol. 29 , no. 12, pp. 6836-6846, Dec 2014.

[24] — - "Performance of multistep finite control set model predictive control for power electronics," IEEE Trans. Power Electron., vol. 30, no. 3, pp. 1633-1644, March 2015.

[25] G. Goodwin, S. Graebe, and M. Salgado, Control system design. Englewood Cliffs, NJ: Prentice-Hall, 2000.

[26] R. P. Aguilera, R. Baidya, P. Acuna, S. Vazquez, T. Mouton, and V. G. Agelidis, "Model predictive control of cascaded H-bridge inverters based on a fast-optimization algorithm," in Proc. 41st Annu. Conf. IEEE Ind. Electron. IECON'15, Yokohama, Japan, Nov. 2015, pp. $004003-$ 004008 .

[27] D. E. Quevedo, R. P. Aguilera, and T. Geyer, "Predictive Control in Power Electronics and Drives: Basic Concepts, Theory, and Methods," in Studies in Computational Intelligence. New York, NY, USA: Springer, Jan. 2014, pp. 181-226.

[28] K. Ilves, L. Harnefors, S. Norrga, and H. P. Nee, "Predictive sorting algorithm for modular multilevel converters minimizing the spread in the submodule capacitor voltages," IEEE Trans. Power Electron., vol. 30, no. 1 , pp. 440-449, 2015.

[29] C. D. Townsend, T. J. Summers, and R. E. Betz, "Phase-Shifted Carrier Modulation Techniques for Cascaded H-Bridge Multilevel Converters," IEEE Trans. Ind. Electron., vol. 62, no. 11, pp. 6684-6696, 2015.

[30] G. Goodwin, M. M. Seron, and J. A. de Don, Constrained Control and Estimation: An Optimisation Approach, 1st ed. Springer Publishing Company, Incorporated, 2010

[31] R. P. Aguilera and D. E. Quevedo, "Predictive control of power converters: Designs with guaranteed performance," IEEE Trans. Ind. Informat., vol. 11, no. 1, pp. 53-63, 2015.
[32] _ - "On stability and performance of finite control set MPC for power converters," in Proc. Workshop Predictive Control Elecct. Drives Power Electron. (PRECEDE), Munich, Germany, Oct. 2011, pp. 55-62.

[33] R. P. Aguilera, R. Delgado, D. Dolz, and J. C. Agüero, "Quadratic MPC with $\ell_{0}$-input constraint," in Proc. 19th IFAC World Congr., Cape Town, South Africa, Aug. 2014, pp. 10888-10893.

[34] R. P. Aguilera, U. G, D. R. A, D. D, and A. J. C, "Quadratic model predictive control including input cardinality constraints," IEEE Trans. Automat. Control, vol. PP, no. 99, pp. 1-1, 2017.

[35] B. Hassibi and H. Vikalo, "On the sphere-decoding algorithm I. Expected complexity," IEEE Trans. Sign. Process., vol. 53, no. 8, pp. 28062818, Aug 2005.

[36] H. Vikalo and B. Hassibi, "On the sphere-decoding algorithm II. Generalizations, second-order statistics, and applications to communications," IEEE Trans. Sign. Process., vol. 53, no. 8, pp. 2819-2834, Aug 2005.

[37] R. B. Schnabel and E. Eskow, "A new modified cholesky factorization," SIAM J. Sci. and Stat. Comput., vol. 11, no. 6, pp. 1136-1158, 1990.

[38] R. A. Horn and C. R. Johnson, Matrix Analysis. Cambridge, U.K.: Cambridge Univ. Press, 1985.

[39] M. Grotschel, L. Lovasz, and A. Schriver, Geometric Algorithms and Combinatorial Optimization. New York: Springer-Verlag, 1993.

[40] S. Boslaugh, "The Pearson Correlation Coefficient," in Statistics in a Nutshell, 2nd ed., 2012, ch. 7.

[41] P. Cortes, J. Rodriguez, C. Silva, and A. Flores, "Delay Compensation in Model Predictive Current Control of a Three-Phase Inverter," IEEE Trans. Ind. Electron., vol. 59, no. 2, pp. 1323-1325, Feb 2012.

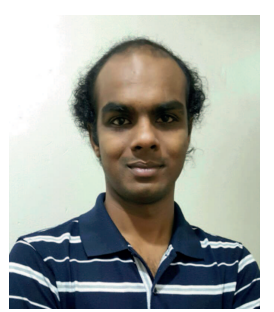

Roky Baidya (S'14) was born in Chittagong, Bangladesh, in 1989. He received his B.Sc. degree in Electrical and Electronic Engineering from the Chittagong University of Engineering and technology (CUET), Chittagong-4349, Bangladesh, in 2011. He is currently pursuing his Ph.D. degree in the School of Electrical Engineering and Telecommunications at the University of New South Wales (UNSW), Sydney, NSW 2052, Australia.

His research interests focus on the model predictive control of power converters and electric drives, including multistep prediction horizon implementation with a fast optimization algorithm, which is, in fact, the prime subject toward the Ph.D. degree.

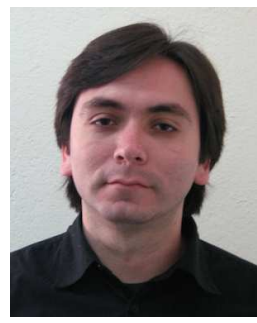

Ricardo P. Aguilera (S'01-M12) received his B.Sc. degree in electrical engineering from the Universidad de Antofagasta, Chile, in 2003; his M.Sc. degree in electronics engineering from the Universidad Tecnica Federico Santa Maria (UTFSM), Chile, in 2007; and his Ph.D. degree in Electrical Engineering from The University of Newcastle (UoN), Australia, in 2012. From 2012 to 2013, he was a Research Academic at UoN, where he was part of the Centre for Complex Dynamic Systems and Control. From 2014 to 2016, he was a Senior Research Associate at The University of New South Wales (UNSW), Australia, where he was part of the Australian Energy Research Institute (AERI). Since Sep 2016, he is with the School of Electrical, Mechanical and Mechatronic Systems, at the University of Technology Sydney (UTS), Australia, where he currently holds a Lecturer position. His main research interests include power electronics, renewable energy, and theoretical and practical aspects on model predictive control. 


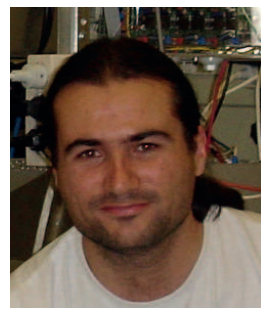

Sergio Vazquez Sergio Vazquez(S'04, M'08, SM'14) was born in Seville, Spain, in 1974. He received the M.S. and $\mathrm{PhD}$ degrees in industrial engineering from the University of Seville (US) in 2006, and 2010, respectively. Since 2002, he is with the Power Electronics Group working in $\mathrm{R} \& \mathrm{D}$ projects. He is an Associate Professor with the Department of Electronic Engineering, US. His research interests include power electronics systems, modeling, modulation and control of power electronics converters applied to renewable energy technologies. Dr. Vazquez was recipient as coauthor of the 2012 Best Paper Award of the IEEE Transactions on Industrial Electronics and 2015 Best Paper Award of the IEEE Industrial Electronics Magazine. He is involved in the Energy Storage Technical Commiteee of the IEEE industrial electronics society and is currently serving as an Associate Editor of the IEEE Transactions on Industrial Electronics.

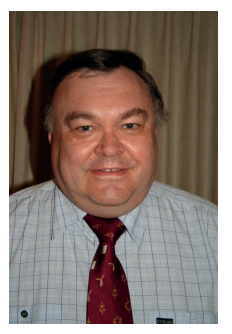

Hendrik du Toit Mouton (S'98-M'00) received the B.Sc, B.Sc.Hons, M.Sc., and Ph.D, in mathematics degrees from the University of the Orange Free-state in 1986, 1987, 1988 and 1991, respectively and the B.Eng and Ph.D. degrees in Electrical Engineering from the University of Stellenbosch in 1996 and 2000 , respectively. He is currently a Professor in electrical Engineering at the University of Stellenbosch and leader of the Power Electronics Research Group. He authored and co-authored more than 130 journal and conference papers in mathematics and power electronics. His research interests include multilevel converters, modelling and control of power electronic converters and class-D audio amplifiers.

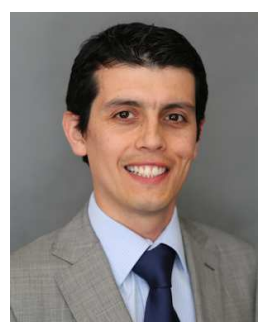

Pablo Acuña (M12) received the B.Sc. degree in electronics engineering, the B.Eng. degree in electronics engineering, and the Ph.D. degree in electrical engineering from the University of Concepcion, Concepcion, Chile, in 2004, 2007, and 2013, respectively.

He is currently a Research Associate in the School of Electrical Engineering and Telecommunications, The University of New South Wales, UNSW Sydney, NSW, Australia. His research interests include electrical power conversion systems and their applications in industry, transportation, and utility. 